\title{
A fast microchannel plate-scintillator detector for velocity map imaging and imaging mass spectrometry
}

\author{
B. Winter, ${ }^{1}$ S. J. King, ${ }^{1}$ M. Brouard, ${ }^{2, a)}$ and C. Vallance ${ }^{1, b)}$ \\ ${ }^{1}$ Department of Chemistry, Chemistry Research Laboratory, University of Oxford, 12 Mansfield Rd, \\ Oxford OX1 3TA, United Kingdom \\ ${ }^{2}$ Department of Chemistry, Physical and Theoretical Chemistry Laboratory, University of Oxford, \\ South Parks Rd, Oxford OX1 3QZ, United Kingdom
}

(Received 31 October 2013; accepted 9 February 2014; published online 28 February 2014)

\begin{abstract}
The time resolution achievable using standard position-sensitive ion detectors, consisting of a chevron pair of microchannel plates coupled to a phosphor screen, is primarily limited by the emission lifetime of the phosphor, around 70 ns for the most commonly used P47 phosphor. We demonstrate that poly-para-phenylene laser dyes may be employed extremely effectively as scintillators, exhibiting higher brightness and much shorter decay lifetimes than $\mathrm{P} 47$. We provide an extensive characterisation of the properties of such scintillators, with a particular emphasis on applications in velocity-map imaging and microscope-mode imaging mass spectrometry. The most promising of the new scintillators exhibits an electron-to-photon conversion efficiency double that of P47, with an emission lifetime an order of magnitude shorter. The new scintillator screens are vacuum stable and show no signs of signal degradation even over longer periods of operation. () 2014 AIP Publishing LLC. [http://dx.doi.org/10.1063/1.4866647]
\end{abstract}

\section{INTRODUCTION}

The first scintillator was developed in 1903, after Sir William Crookes ${ }^{1}$ peered into the eyepiece of his microscope and made an astonishing discovery: after accidentally spilling radium particles onto a zinc sulphide screen he observed discrete light flashes, stimulated by the emission of single alpha particles from the radium. Today, fast and efficient scintillator screens are in considerable demand for a wide range of particle time-of-flight imaging applications in fields such as highenergy ion physics, mass spectrometry, and chemical reaction dynamics. ${ }^{2}$ The optimum characteristics of a scintillator vary with application, but generally include a suitable emission wavelength and emission lifetime, and a high brightness, characterised by the efficiency of energy conversion from the kinetic energy of the incident particle to the emitted photons.

For the remainder of this article, we focus our attention primarily on applications in velocity-map imaging and microscope-mode imaging mass spectrometry, in which scintillator screens are used to detect few-keV electrons generated following charged particle impact on a microchannel plate ion/electron detector. In such experiments, ions or electrons from the process of interest are accelerated along a flight tube to the microchannel plates (MCPs), which amplify each incident particle into a burst of around $10^{6}$ electrons. These electrons are accelerated towards a scintillator screen, generating an optical image of the incident ion distribution, which may then be captured by a CCD or CMOS camera. The scintillators most commonly used in ion detectors of this type are $\mathrm{P} 47\left(\mathrm{Y}_{2} \mathrm{SiO}_{5}: \mathrm{Ce}\right)$ or $\mathrm{P} 43\left(\mathrm{Gd}_{2} \mathrm{O}_{2} \mathrm{~S}: \mathrm{Tb}\right)$ phosphors. These inorganic, solid-state materials show a high emission efficiency in the near-UV and visible regions of the spectrum, which over-

\footnotetext{
a)Electronic mail: mark.brouard@chem.ox.ac.uk

b) Electronic mail: claire.vallance@chem.ox.ac.uk
}

laps with the sensitive range of wavelengths for most CCD cameras. A typical emission lifetime for a P47 screen is about $70 \mathrm{~ns}$, while the P43 screen emits for more than $1 \mathrm{~ms}^{2}{ }^{2}$ While faster phosphors are available for time-of-flight applications, they tend to suffer from greatly reduced brightness relative to P47, often to the point where the signal-to-noise ratio either becomes insufficient to achieve high resolution imaging, or in the worst cases, insufficient to detect particles at all. The emission characteristics of a number of commonly used scintillators are given in Table I.

The emission lifetime of a $\mathrm{P} 47$ phosphor screen is sufficient to achieve sub-Dalton mass resolution in velocity-map (or spatial-map) imaging studies for ions in the sub-100 Da range. In most experiments of this type, the time-of-flight (and therefore mass) resolution is also limited considerably by the intrinsically poor mass focusing properties of the velocitymap imaging ion optics. However, the recent development of improved ion optics has raised the possibility of carrying out imaging studies with high mass resolution on significantly larger molecules, and faster scintillator screens are now needed in order to realise these measurements. ${ }^{3,4}$ For example, in microscope-mode imaging mass spectrometry, using the post-extraction differential acceleration method, ${ }^{5}$ Brouard et $\mathrm{al}^{4}{ }^{4}$ achieved an ion-optics-limited mass resolution of $m / \Delta m=2000$, but the high time-of-flight resolution was lost during image acquisition due to the slow response time of the P47 phosphor used in the position-sensitive ion detector. While the limitations imposed by slow phosphor response times can be circumvented, in part, by employing alternative detectors such as the TimePix sensor ${ }^{6-9}$ or delay line detectors, ${ }^{10,11}$ there are considerable advantages to using scintillator screens. A MCP/scintillator detector may be used to detect large numbers of particles simultaneously, and is easily coupled with a CCD or CMOS camera for image capture, or with a photomultiplier tube (PMT) or photodiode in order 
TABLE I. Properties of organic and inorganic scintillators, taken from Ref. 2.

\begin{tabular}{|c|c|c|c|c|}
\hline Scintillator & Type & $\begin{array}{l}\text { Max. emission } \\
\text { wavelength } \\
\text { in } \mathrm{nm}\end{array}$ & $\begin{array}{c}\text { Emission } \\
\text { efficiency } \\
\text { relative } \\
\text { to anthracene }\end{array}$ & $\begin{array}{c}\text { Emission } \\
\text { lifetime }\end{array}$ \\
\hline Anthracene & Organic crystal & 447 & 100 & $30 \mathrm{~ns}$ \\
\hline BC-404/EJ-204 & Plastic & 423 & 65 & $2.4 \mathrm{~ns}$ \\
\hline BC-408/EJ-200 & Plastic & 425 & 64 & $2.1 \mathrm{~ns}$ \\
\hline P22 & Inorganic & 530 & $1631-1755$ & $>1 \mathrm{~ms}$ \\
\hline P43 (YAG) & Inorganic & 545 & $483-725$ & $>1 \mathrm{~ms}$ \\
\hline P47 & Inorganic & 430 & $145-156$ & $70 \mathrm{~ns}$ \\
\hline YAP & Inorganic & 370 & $109-117$ & $27 \mathrm{~ns}$ \\
\hline
\end{tabular}

to monitor the total light intensity and thereby record timeof-flight spectra. Fast intelligent pixel sensors, such as the PImMS (pixel imaging mass spectrometry) multimass imaging sensor ${ }^{12-16}$ are capable of achieving considerably better time resolution than conventional CCD cameras, and this has driven us to develop faster scintillators in order that the scintillator does not impose the primary limitation on time-of-flight resolution.

As shown in Table I, a number of scintillators are available with shorter emission lifetimes than a P47 phosphor. In particular, plastic scintillators, which generally contain anthracene derivatives locked into a polymer matrix in order to confer vacuum stability, commonly have emission lifetimes of a few nanoseconds. Unfortunately, however, their speed usually comes at the cost of considerably reduced brightness. Plastic scintillators, e.g., BC-404, are often used in time-offlight detector assemblies, converting electrons from a microchannel plate detector into an optical signal which can be detected by a PMT or photodiode as an alternative to recording the electron current directly from the MCPs. The low brightness requires the detector assembly to be operated at very high gain in order to achieve sensitive ion detection. This not only increases the risk of arcing across the detector components, but space charge repulsion between the electrons generated from each ion burst leads to a spreading out of the resulting "bright spot" on the phosphor, and a corresponding reduction in image resolution. While these effects can be mitigated to some extent by employing event counting algorithms, the ability to run the MCPs at lower gains is very helpful when attempting to optimise image quality.

Other organic scintillators have also been investigated for applications in mass spectrometry. For example, Dubois, Zenobi, and co-workers ${ }^{17,18}$ used the organic components But-PBD (2-(4-tert-Butylphenyl)-5-(4biphenylyl)-1,3,4-oxidiazole) and BBOT (2,5-bis(5'-tertbutyl-2-benzoxazolyl)thiophene) to build an ion-to-photon conversion detector (IPD). The detector consisted of a glass plate coated with a layer of the organic material and mounted in front of a PMT. The unit was found to have a time resolution of $4 \mathrm{~ns}$ when detecting $24 \mathrm{keV}$ ions, and could detect ions with masses up to $150 \mathrm{kDa}$. In later work, the introduction of a conversion dynode mounted in front of the BBOT scintillator plate improved the signal levels further, to the point where they were comparable with those obtained using a BC-404 plastic scintillator. ${ }^{19}$

While there has been very little work on the emission properties of organic compounds in the solid phase following collisional excitation, a number of organic materials have been characterised for their photoemission properties following absorption of light, and we review the results of these studies here. Organic emitters generally employ aromatic hydrocarbon compounds, in which transfer of kinetic energy from an incoming particle excites the delocalised $\pi$ electrons into higher lying electronic states. ${ }^{2,20}$ The initially excited states decay rapidly via internal conversion processes, until they occupy primarily the $v=0$ vibrational levels of the lowest lying singlet and triplet electronic states, from which emission occurs. The energy separation between the ground state and the lowest singlet state is typically $3-4 \mathrm{eV}$, with the emission spectrum of the scintillator being determined by the states from which fluorescent emission occurs and the vibrational levels of the ground state that lie within the FranckCondon envelope. Emission from the lowest lying triplet state is spin forbidden, and occurs over a much longer lifetime; in order to obtain a fast scintillator response, it is therefore desirable to identify or design compounds in which the population of these triplet states is minimised.

A broad range of organic compounds have been evaluated previously as potential scintillator materials following photoexcitation. For example, in the 1950s, Sangster and Irvine $^{21}$ analysed the scintillation properties of 55 pure crystalline compounds following excitation by ultraviolet light and by ${ }^{60} \mathrm{Co} \gamma$ radiation. Amongst others, para-terphenylene and para-quaterphenylene were identified as efficient scintillators. These molecules share the feature that all carbon atoms are $s p^{2}$ hybridised, allowing the $\pi$ electron density to be delocalised over the entire molecule. In addition, in solution the phenyl units can undergo free rotation along the molecular axis, allowing the molecule to crystallise in lowenergy conformations in which steric conflicts between the substituents located ortho and meta to the adjacent phenyl ring(s) are minimised. Sangster and Irvine ${ }^{21}$ determined that both of these properties are beneficial in generating an efficient scintillator. Nowadays, poly-para-phenylene derivatives are used widely as fluorescent laser dyes. Molecular structures for four common laser dyes are shown in Figure 1. Exalite 384, Exalite 389, and Exalite 398 are para-quaterphenylenes, while Exalite 404 is a para-quintphenylene. The molecular structures have been optimised to maximise the performance of the compounds as fluorescent dyes; we see that relative to the parent hydrocarbons, the terminal hydrogen atoms in the Exalite 389, 398, and 404 molecules have been substituted either with ether or amyl groups, and methylene bridges have been introduced in all dyes in the meta or ortho position between adjacent phenyl rings. The effects of both of these substitutions have been the subject of a number of studies: for example, the effect of ring bridging on the optical properties following photoexcitation at $308 \mathrm{~nm}$ was investigated by Rinke and co-workers. ${ }^{22}$ It was established that the bridging, which forces two adjacent phenyl rings into one plane, shifts the emission spectrum towards longer wavelengths, with a methylene bridge conferring optimum photostability. 


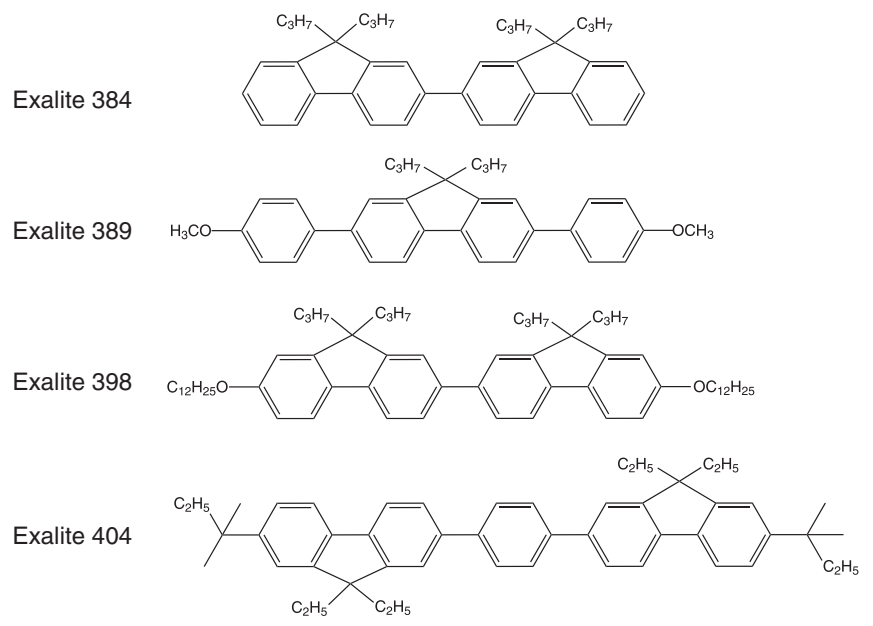

FIG. 1. The molecular structures of the laser dyes employed in this study.

Ethylene or ether bridges resulted either in a reduced photonto-photon conversion efficiency or a reduced damage threshold. It was found that the photoemission output could be increased further by adding two propyl groups to the methylene bridge. $^{23}$ The substitution of the terminal hydrogen atoms with alkoxy groups was generally found to red-shift the emission spectrum, ${ }^{24}$ and for singly bridged molecules was also reported to enhance the photon to photon conversion efficiency of the dye. ${ }^{25}$ Kauffman et al. ${ }^{23}$ found that the alkoxy substitution reduced the photostability of the compound, but Fleitz and co-workers ${ }^{26}$ later reported that terminal tert-butyl substitution increases the stability. Inamdar ${ }^{27}$ measured the fluorescent emission lifetime for two alkyl and alkyloxy substituted para-quarterphenyl molecules, and found the lifetime to be virtually independent of the terminal substituent. An emission lifetime of $\sim 1$ ns was measured for Exalite 398 in solution.

Recently, Merz and co-workers ${ }^{28}$ presented a theoretical model to predict the effect of the various substitutions on the photophysical properties of the poly-para-phenylenes. For example, shifts in the emission wavelength were predicted as function of the Hammett parameter of the terminal substitution groups. The predictions of the model were found to be in good agreement with the experimentally determined photoemission properties.

To our knowledge, there is only one previous report of a poly-para-phenylene dye having been employed in a particle detector. Tymianski and Walker ${ }^{29}$ patented a scintillator system for the detection of high energy particles, such as X-rays, $\gamma$-rays, $\beta$-particles, $\alpha$-particles, and thermal neutrons. The detector was fabricated from a mixture of $92 \%$ organic polymer, $5 \%$ polycyclic compound such as substituted naphthalene, and up to $3 \%$ of a fluorophore, for example, Exalite 389. Energy deposited into the organic polymer by the penetrating radiation is transferred to the polycyclic organic compound, which emits light upon relaxation. The emitted photons are absorbed by the Exalite dye, and the resulting fluorescence forms the detected signal. This arrangement differs from that described in the present work, in that the Exalite dye acts as a wavelength shifter, rather than being excited directly by kinetic energy transfer from incident particles.
As the above discussion shows, the emission properties of the Exalite dyes following photoexcitation have been well studied and characterised. In the present work, we evaluate the emission properties of the dyes following excitation via charged-particle impact, and compare their performance with commonly used inorganic scintillators. In order to select the most effective scintillator for use in ion imaging applications, a series of poly-para-phenylene dyes, widely available under the trade names Exalite 384, Exalite 389, Exalite 398, and Exalite 404, have been tested in a MCP-based ion imaging detector and compared with the $\mathrm{P} 47$ inorganic scintillator. The key comparators are the emission lifetime, emission spectrum, conversion efficiency, stability, and spatial resolution of the scintillator screens.

\section{EXPERIMENTAL}

\section{A. Scintillator screen preparation}

The substrates for all of the scintillator screens described in this work were quartz slides of thickness $1.1 \mathrm{~mm}$, coated with a layer of the transparent conducting compound indium tin oxide (ITO). Square $(25 \mathrm{~mm})$ and circular $(50 \mathrm{~mm}$ diameter) substrates were used. Inorganic phosphors were deposited using a sedimentation process, while organic dye screens were prepared via vacuum sublimation.

The sedimentation process employed was similar to that used to produce phosphor screens in small-scale commercial operations. Clean ITO-coated substrates were placed in a tilted beaker $\left(\sim 8^{\circ}\right)$ with the conductive side facing upwards. Barium chloride solution (1.16 mM in deionised water) was added to the beaker until the plates were submerged to a depth of $5 \mathrm{~cm}$. A mixture of $0.15 \mathrm{~g}$ of P47 phosphor powder (Phosphor Technology, UK, $95 \%$ of particles $<7.2 \mu \mathrm{m}$ ) in $20 \mathrm{ml}$ of $0.37 \mathrm{M}$ potassium silicate solution was prepared, stirred thoroughly, and poured slowly through a sieve to form a suspension mixture within the beaker. After a sedimentation period of $20 \mathrm{~min}$, the remaining solution was syphoned from the beaker, taking care to avoid generating turbulence which might damage the screens. The newly prepared screens were dried in an oven for $20 \mathrm{~min}$ at $60{ }^{\circ} \mathrm{C}$ prior to installation within an imaging detector. Typical phosphor thicknesses on screens prepared using this method were around $350 \mu \mathrm{m}$.

A photograph of a $\mathrm{P} 47$ phosphor screen prepared according to the sedimentation protocol is shown in Figure 2(a). Some inhomogeneities in the screen surface are visible, caused either by turbulence during solvent removal or (a)

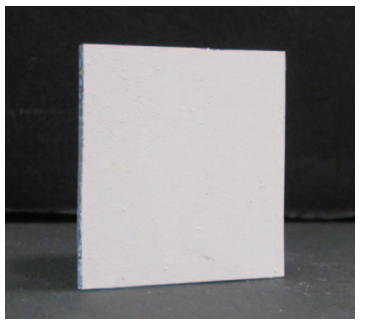

(b)

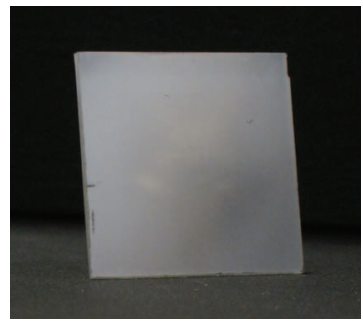

FIG. 2. (a) A typical P47 scintillator screen prepared by sedimentation; (b) a typical organic dye screen prepared by vacuum sublimation. Both screens are prepared on $25 \mathrm{~mm}$ ITO-coated glass substrates. 
air bubbles trapped during the sedimentation step. However, large enough areas of blemish-free screen can be prepared to match the field of view of the detector.

To prepare organic dye screens by vacuum sublimation, a quantity of dye $(0.05 \mathrm{~g}-0.15 \mathrm{~g})$ was placed in the base of the sublimation apparatus (Ace vacuum sublimation apparatus, $60 \mathrm{~mm}$ internal diameter). A clean ITO plate was mounted, coated-side-down, on the cold finger of the apparatus, which was then sealed and evacuated to a pressure of 14 mbar. The cold finger was filled with a water/ice slush, and a temperature ramp was applied to the sublimation chamber using a hot plate. Depending on the material, the dye crystals either melted and evaporated, or sublimed, and subsequently crystallised onto the ITO plate. Care was taken to ensure complete evaporation/sublimation of the dye sample in order to achieve reproducible scintillator screen thicknesses of around $200 \mu \mathrm{m}$. A typical scintillator screen prepared using Exalite 404 dye is shown in Figure 2(b).

The scintillator screens prepared according to one or other of the procedures described above were mounted on a vacuum flange in between a chevron-pair of $40 \mathrm{~mm}$ microchannel plates and a $60 \mathrm{~mm}$ viewport. The spacing between the MCPs and the phosphor was $1.5 \mathrm{~mm}$. Electrical feedthroughs mounted in the flange allowed control of the potentials applied to the MCPs and scintillator screen. For some of the calibration and comparison measurements described below, composite screens were fabricated using smaller sections of several different scintillators.

No detailed data are available on the vapour pressures of the Exalite dyes, with the manufacturer's specifications simply stating it as "negligible" at room temperature. However, since installation of the new detector did not yield any noticeable difference in pump-down time or achievable base pressure within our vacuum system when compared with a commercial P47-based detector, we do not believe there to be any outgassing or vapour-pressure-related problems with the new materials, at least down to pressures in the $10^{-8}$ mbar range.

\section{B. Screen characterisation}

All particle imaging experiments carried out to characterise the detectors were performed using an existing velocitymap imaging spectrometer, which has been described in detail previously. ${ }^{30}$ Briefly, a molecular beam of methyl iodide $\left(\mathrm{CH}_{3} \mathrm{I}\right)$ or carbonyl sulphide (OCS), prepared by seeding $5 \%$ of the relevant gas in 1 bar helium, was generated via supersonic expansion through a pulsed valve (General valve series 10). The beam passed through a $1 \mathrm{~mm}$ skimmer and entered the three-electrode velocity-map imaging ion optics, consisting of repeller, extractor, and ground electrodes. The $2 \mathrm{~mm}$ thick electrodes are $90 \mathrm{~mm}$ in diameter, with apertures of $1 \mathrm{~mm}, 20 \mathrm{~mm}$, and $20 \mathrm{~mm}$, respectively, and are spaced by $15 \mathrm{~mm}$. The molecular beam was intersected midway between the repeller and extractor electrodes by a focused laser beam (details to follow), and the resulting ions or electrons were accelerated and velocity-mapped through a $730 \mathrm{~mm}$ flight tube onto the imaging detector.
Electrons generated by $118 \mathrm{~nm}$ laser-induced photodissociation and ionization of methyl iodide provided a suitably fast signal to characterise the time resolution of the ion detector. The $118 \mathrm{~nm}$ laser pulse was generated by focusing the third harmonic of a Nd:YAG laser (Continuum Surelite I) into a gas cell filled with a phase-matched mixture of 24 mbar of $\mathrm{Xe}$ and $325 \mathrm{mbar}$ of $\mathrm{Ar}^{31}{ }^{31}$ In order to irradiate the detector uniformly with electrons, these measurements were not carried out under velocity mapping conditions. Instead, the repeller electrode was held at a potential of $-2 \mathrm{kV}$, and the extractor and ground plates were fixed at ground potential. The voltages applied across the MCP stack and between the back MCP and the scintillator screen were adjusted in order to achieve sufficient signal levels to determine the rise and decay time of the signal, as well as the lifetime of the emission from the scintillator. The time-dependent signal from the phosphor was recorded using a photomultiplier tube (Hamamatsu H678020) coupled to a digital oscilloscope (LeCroy DDA-260). The resulting signals were accumulated on a PC over 100 timeof-flight cycles. The minimum width of the electron time-offlight peak in this configuration is limited primarily by the laser pulse duration.

The emission spectra of the various scintillators following electron excitation were determined by mounting a monochromator coupled to a PMT in front of the scintillator screen. Again, the detector was illuminated uniformly by electrons, this time by operating with a $-6.0 \mathrm{kV}$ potential on the repeller electrode of the ion optics assembly, with the remaining two electrodes grounded. The detector was biased with $1.3 \mathrm{kV}$ across the MCPs and $3.55 \mathrm{kV}$ on the phosphor. The signal levels were optimised for each scintillator by adjusting the widths of the monochromator's input and output slits. The maximum slit width employed resulted in a wavelength resolution better than $5 \mathrm{~nm}$. Emission spectra were acquired by recording the integrated signal within the electron ToF peak as the monochromator wavelength was scanned, with due consideration of the wavelength dependent sensitivity curve of the PMT and the light absorption of the ITO coated glass plate. For comparison, the emission spectrum of an Exalite 404 screen was measured following photoexcitation at $337 \mathrm{~nm}$ using a Fluoromax 4 emission spectrometer based at the Rutherford Appleton Laboratory. Dye emission spectra were also recorded in dichloromethane solution following photoexcitation at $355 \mathrm{~nm}$.

The absolute electron-to-photon conversion efficiency is a relatively difficult parameter to measure in our imaging spectrometer, since the number of detected ions or electrons depends on a number of different experimental parameters, and the photomultipliers and cameras available to us do not provide absolute photon counts. However, by integrating ion or electron time-of-flight peaks detected with different scintillator screens under the same operating conditions, a relative value for the electron-to-photon conversion efficiency can be determined for each scintillator. In practice, this was achieved by fabricating a segmented screen in which two quadrants were coated in $\mathrm{P} 47$ phosphor and two with the scintillator of interest, with the potentials applied to each segment able to be varied independently. This arrangement allowed the performance characteristics of each screen to be compared 
directly with those of a P47 screen, and also allowed the relative performance of both the phosphor under study and P47 to be compared at different excitation energies. Typically, a potential of $1.1 \mathrm{kV}$ was applied across the MCPs. One of each pair of scintillators was held at $2.5 \mathrm{kV}$, while the potential applied to the second of each pair was incremented in steps of $100 \mathrm{~V}$ up to a maximum of $4.0 \mathrm{kV}$ relative to ground. Under these operating conditions, the detector was used to record velocity-map images of the iodine fragments formed during the $245.2 \mathrm{~nm}$ photolysis of methyl iodide.

The stability of the screens over extended operating periods was also investigated in order to determine whether their performance degrades due to cumulative damage. For these measurements, a reference P47 scintillator screen was mounted in the detector beside an Exalite 404 screen, and the detector was bombarded continuously with electrons for $47 \mathrm{~h}$ with $1.1 \mathrm{kV}$ across the MCPs and $2.5 \mathrm{kV}$ on the scintillator screens. The resulting signals were monitored using a CCD camera. Around 600 events were recorded per time-of-flight cycle across both scintillator screens, at a repetition rate of $10 \mathrm{~Hz}$, yielding an incident electron current on the detector of $\sim 6000$ electrons per second. Assuming a typical MCP gain of $\sim 5 \times 10^{5}$, this corresponds to an incident electron current on the scintillator of around $3 \times 10^{9}$ electrons per second, or 5 $\times 10^{14}$ electrons in total over the course of the measurement.

Finally, the spatial resolution of the screens was investigated through velocity-map ion imaging of $\mathrm{CO}^{+}$ions formed following $230 \mathrm{~nm}$ photolysis of OCS. These measurements were carried out with the newly tested scintillator MCP detector and with a commercial MCP/P47 imaging detector (Burle APD 3075FM). The ion images were collected using a CCD camera and the observed signals were thresholded and centroided to optimise the image resolution.

\section{RESULTS AND DISCUSSION}

The key performance parameters of the new scintillator screens are their time resolution, brightness, emission spectra, stability, and spatial imaging properties. These will be explored in turn in the following.

\section{A. Time resolution}

Figure 3 shows electron time-of-flight peaks recorded using MCP/scintillator detectors employing Exalite 404, Exalite 389, and P47 phosphor screens. It is immediately obvious from the data that the dye-based screens offer greatly improved time resolution relative to the $\mathrm{P} 47$ phosphor. The emission lifetime of each scintillator can be fitted well to a single exponential function of the form

$$
I=I_{0} e^{-t / \tau},
$$

where $I_{0}$ is the peak emission intensity and $\tau$ is the emission lifetime, which has a characteristic value for each scintillator. As an alternative to the emission lifetime, the decay time of the luminescence emitted from a scintillator is often reported in terms of the time taken for the light intensity to fall to $10 \%$ of its peak value. In Table II, we list the emission lifetime (i.e., the time constant of the exponentially decaying signal),

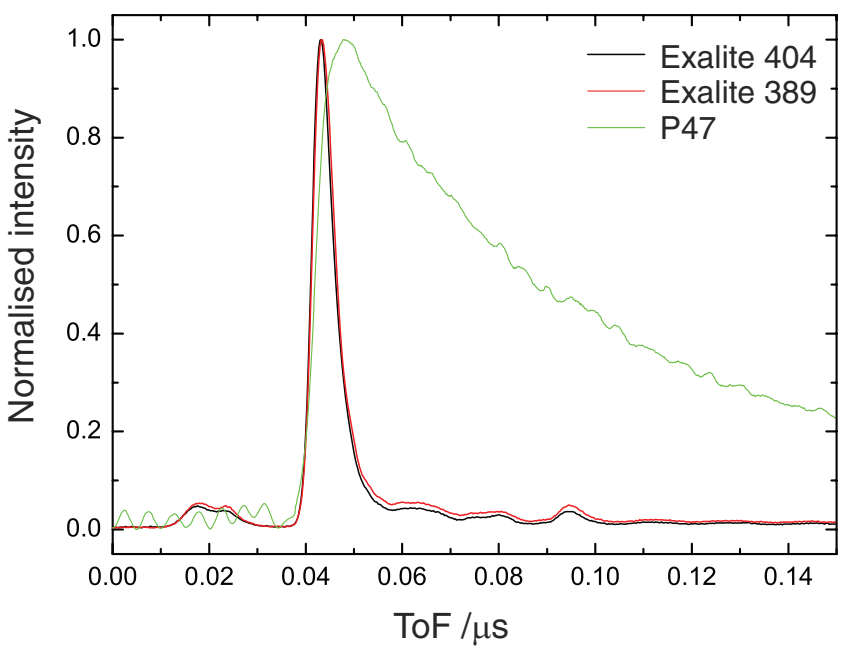

FIG. 3. Electron time-of-flight signal recorded for the Exalite 404, Exalite 389 and P47 screens.

rise time (from $10 \%$ to $100 \%$ of peak intensity), and fall time (from $100 \%$ to $10 \%$ of peak intensity) of the signals measured for each of the scintillator screens studied. The reported values are useful for comparing the performance of different scintillators in a charged particle detector exposed to identical electron pulses. However, we note that the measured time resolutions are almost certainly limited either by the $3-5 \mathrm{~ns}$ duration of the laser pulse used to generate the electrons or by the mass-focusing capabilities of the ion optics in the imaging spectrometer. These are difficult to deconvolute from the measured signals, as this would require an independent method of measuring the electron flux impinging on the scintillator screen. However, SIMION ${ }^{32}$ simulations of the electron trajectories through our ion optics system, based on a realistic model for the electron spatial and velocity distributions generated within our experimental setup, predict an arrival time spread of around $4 \mathrm{~ns}$, consistent with our shortest measured emission lifetimes (for Exalite 389 and 404) of $3.7 \mathrm{~ns}$.

The values listed in Table II therefore represent an upper limit to the true emission lifetimes of the screens. Of the various scintillators studied, Exalite 389 and Exalite 404 dyes exhibit the fastest time response, with measured emission lifetimes of less than $4 \mathrm{~ns}$, more than an order of magnitude faster than the P47 phosphor most commonly used in commercial position-sensitive ion detectors. Based on these results, the

TABLE II. Timing parameters determined for the various scintillator screens following detection of a pulse of electrons. $\tau$ represents the emission lifetime determined from a single-exponential fit to the data, $\mathrm{t}_{\text {rise }}$ is the signal rise time from $10 \%$ to $100 \%$ of the peak maximum, and $\mathrm{t}_{\text {fall }}$ describes the signal decay time from $100 \%$ to $10 \%$.

\begin{tabular}{lrcr}
\hline \hline Scintillator & \multicolumn{1}{c}{$\tau(\mathrm{ns})$} & $\mathrm{t}_{\text {rise }}(\mathrm{ns})$ & \multicolumn{1}{c}{$\mathrm{t}_{\text {fall }}(\mathrm{ns})$} \\
\hline P47 & $69.3 \pm 0.8$ & $6.7 \pm 0.2$ & $168 \pm 2.3$ \\
Exalite 384 & $4.7 \pm 0.5$ & $4.2 \pm 0.8$ & $9.3 \pm 1.5$ \\
Exalite 389 & $3.7 \pm 0.5$ & $3.5 \pm 0.6$ & $7.7 \pm 1.0$ \\
Exalite 398 & $9.8 \pm 0.4$ & $5.1 \pm 1.1$ & $26.1 \pm 1.9$ \\
Exalite 404 & $3.7 \pm 0.2$ & $3.5 \pm 0.2$ & $7.7 \pm 0.3$ \\
\hline \hline
\end{tabular}



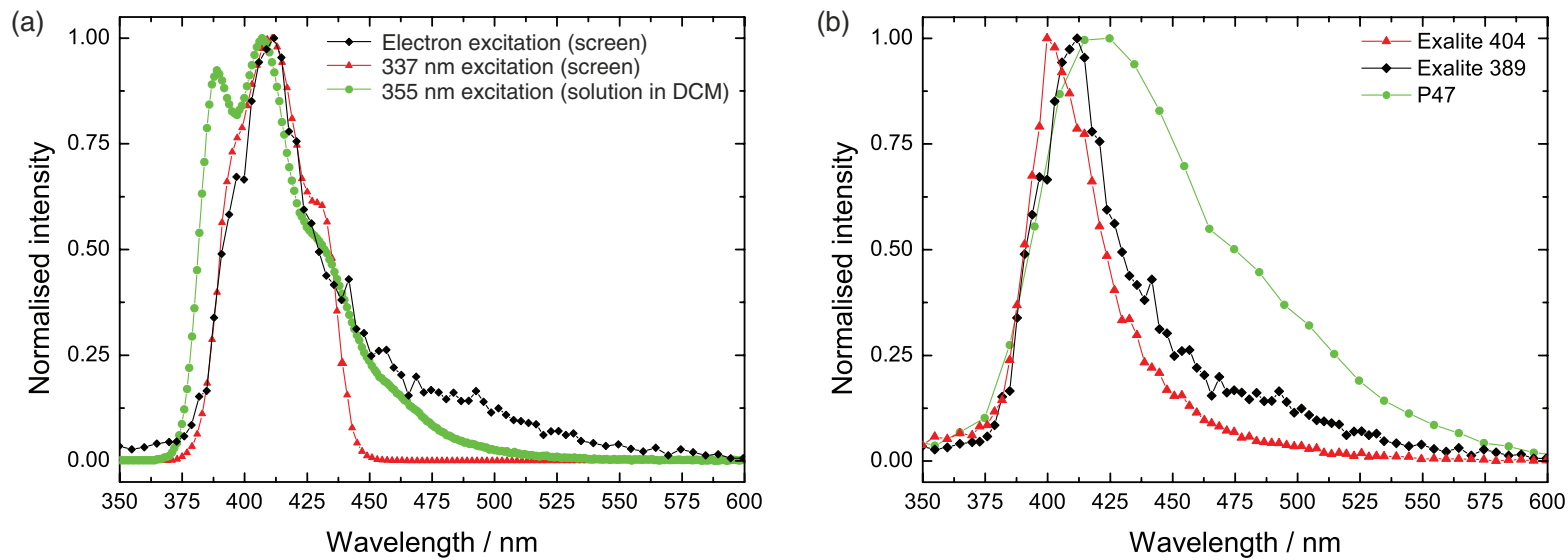

FIG. 4. (a) Emission spectra of Exalite 404 following $337 \mathrm{~nm}$ photoexcitation of a dichloromethane solution, $337 \mathrm{~nm}$ photoexcitation of a scintillator screen, and electron excitation of the scintillator screen; (b) emission spectra of Exalite 404, Exalite 389, and P47 scintillator screens following electron excitation.

remainder of the study focused on further characterisation of the Exalite 389 and Exalite 404 screens.

For the Exalite 404 screen, the emission lifetime following electron excitation was compared with that following photoexcitation in a Fluoromax 4 emission spectrometer. The excitation source for this measurement was a $337 \mathrm{~nm}$ LED. A dual-exponential fit to the signal yielded a primary component with a lifetime of $1.58 \pm 0.03 \mathrm{~ns}$ and a weaker secondary component with a lifetime of $9.62 \pm 0.13 \mathrm{~ns}$. This is of the same order as the emission lifetime measured following electron excitation in the velocity-map imaging experiment.

\section{B. Photoemission spectra}

Besides exhibiting a fast fluorescence lifetime, any scintillator employed in ion imaging experiments must also emit in a wavelength region corresponding to the sensitive range of the CCD or CMOS imaging sensor used for image capture. The emission spectrum following charged-particle excitation is therefore an important characteristic of the screens. It is also interesting to compare the emission spectra of the selected dyes with their solution-phase counterpart, which has been very well characterised as a result of their primary applications as laser dyes.

Figure 4(a) shows emission spectra recorded for Exalite 404 in dichloromethane solution following excitation at 337 $\mathrm{nm}$, and for Exalite 404 scintillator screens following excitation with a $337 \mathrm{~nm}$ LED and via $2.25 \mathrm{keV}$ electron impact in the imaging spectrometer. Following optical excitation, the solution-phase and solid-state emission spectra show a similar rising edge at short wavelengths, but while the solidstate emission exhibits a relatively sharp cutoff at $450 \mathrm{~nm}$, the solution-phase emission has a long-wavelength "tail" extending out to around $525 \mathrm{~nm}$. The emission spectrum recorded following electron excitation again shows a similar rising edge, but exhibits an even longer tail, extending out to around $600 \mathrm{~nm}$. For both types of excitation, the peak emission wavelength of the screen is $412 \mathrm{~nm}$.

The similarities between the three spectra appear to indicate that regardless of which electronic state(s) are initially populated in the excitation process, emission occurs primarily from the same electronic state, presumably following rapid relaxation from higher states via internal conversion processes. When employed in solution in commercial dye lasers, the Exalite dyes emit from their first excited singlet state, ${ }^{33}$ and at least to first order this appears to be the case following either photoexcitation or electron-impact excitation in the solid state.

Initially, it was thought that the long-wavelength tail on the emission spectrum recorded following electron excitation indicated emission from the first excited triplet state. This would not be unexpected, since excitation via charged particle collisions does not obey optical selection rules, and a mixture of singlet and triplet states are likely to be excited in the collision, with internal conversion processes returning population to the lowest excited singlet and triplet state, respectively. However, phosphorescent emission from a triplet state to the ground electronic state is expected to be associated with a much longer emission lifetime than fluorescent emission from a singlet state, and this was not observed to be the case within the signal-to-noise levels achievable in this wavelength region. The tail can perhaps be explained by chargedparticle excitation accessing different regions of the $S_{1}$ potential energy surface which have more favourable FranckCondon overlap with higher vibrational levels of the ground state, but considerable further work would be required in order to reach any definitive conclusions.

Figure 4(b) compares the emission spectra recorded following charged-particle excitation of P47, Exalite 389, and Exalite 404 scintillator screens. All three screens emit across a similar wavelength range, with the two Exalite screens showing very similar emission spectra, and the $\mathrm{P} 47$ phosphor peaking at a slightly longer wavelength. Thus, each scintillator is compatible with silicon-based CCD and CMOS image sensors, though optimum performance will be achieved for sensors optimised for detection at the blue end of the optical spectrum.

\section{Brightness}

The measured brightness of a scintillator screen is determined by the electron-to-photon conversion efficiency. A 
(a)

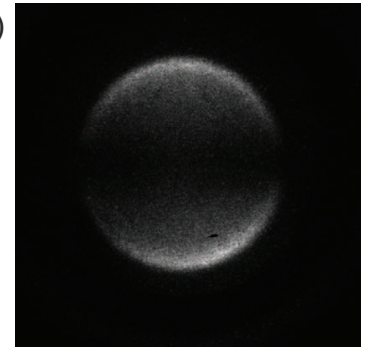

(c)

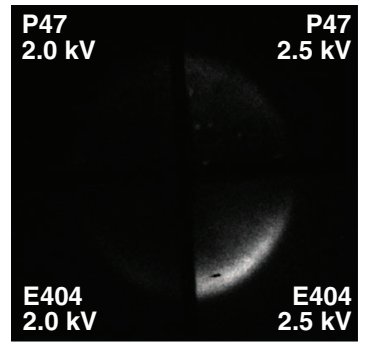

(b)

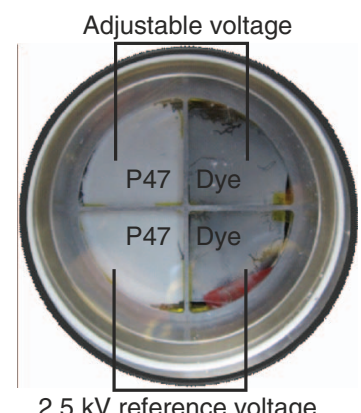

(d)

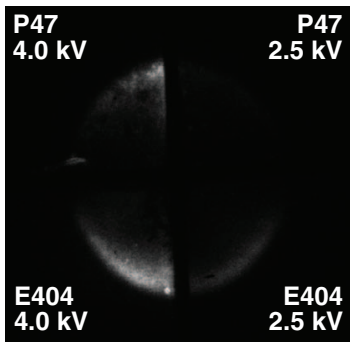

FIG. 5. (a) A typical velocity-map ion image of the iodine fragment formed in the $245.2 \mathrm{~nm}$ photolysis of $\mathrm{CH}_{3} \mathrm{I}$, recorded using a $\mathrm{P} 47$ phosphor screen; (b) typical quadrant detector assembly used for the relative brightness measurements; (c) and (d) representative images obtained using a quadrant detector employing Exalite 404 (E404) and P47, at two different sets of acceleration potentials. The diameter of the composite screen is $45 \mathrm{~mm}$, with an image diameter of $\sim 25 \mathrm{~mm}$.

brighter screen reflects an improved charged particle detection efficiency, thereby reducing data acquisition times, and yields an improved signal-to-noise ratio. As noted in Sec. II B, we are unable to measure the absolute conversion efficiency using our experimental setup. Instead, the brightness of the new scintillators were compared with that of a P47 phosphor screen.

The signal used for comparison between the various screens was ion signal recorded for the $\mathrm{I}^{+}$ion formed in the $245.2 \mathrm{~nm}$ photolysis of $\mathrm{CH}_{3} \mathrm{I}$. Neutral iodine atoms formed in the photodissociation process are ionised by a second photon from the same laser pulse, yielding the detected ions. Iodine atoms are formed with a fairly narrow velocity distribution, yielding a single "ring" in the image, with a $\cos ^{2} \theta$ angular distribution about the laser polarisation axis. A typical velocity-map image detected with a standard dual-MCP detector coupled to a P47 phosphor screen is shown in Figure 5(a).

As noted in Sec. II B, the dye scintillators were compared with the P47 phosphor by fabricating a quadrant scintillator screen in which two quadrants employed P47 and two employed the dye of interest. Such an arrangement also allows the effect of the incident particle energy to be investigated, with one $\mathrm{P} 47 /$ dye pair held at a fixed MCP-scintillator potential difference to provide a reference signal. An example of one such composite screen is shown in Figure 5(b). Two images acquired using the quadrant detector, both employing P47 and Exalite 404 scintillators, but with different applied scintillator potentials, are shown in Figures 5(c) and $5(d)$. Note that in order to demonstrate the relative performances of the scintillator screens making up each of the four detector quadrants, the image brightnesses have been adjusted

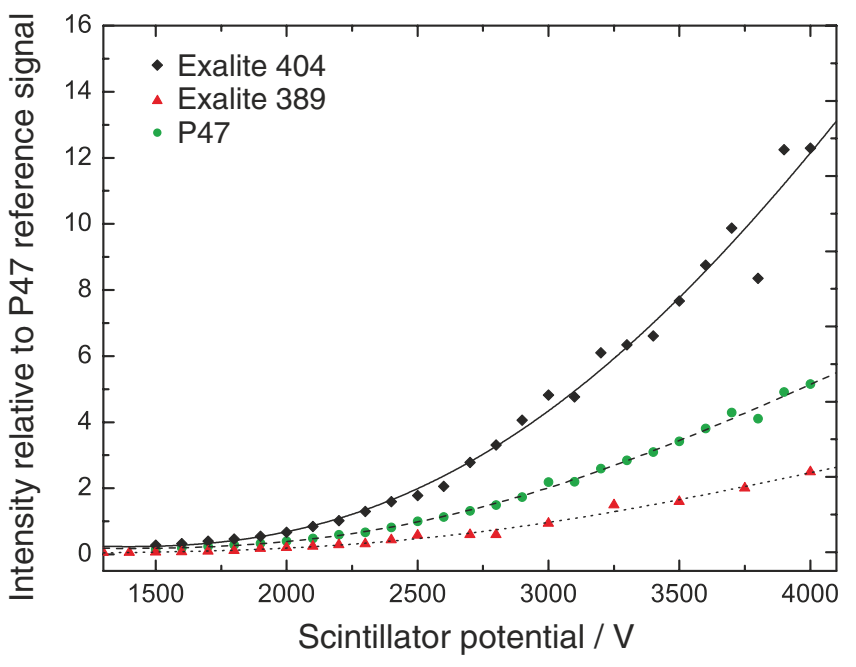

FIG. 6. Relative scintillation efficiencies for the P47, Exalite 389, and Exalite 404 scintillator screens as a function of incident electron energy. Efficiencies are plotted relative to $\mathrm{P} 47$ at an acceleration potential of $2.5 \mathrm{kV}$. Each of the data sets was fitted with a 4th order polynomial: Exalite 404 (solid line), P47 (dashed line), Exalite 389 (dotted line).

in post-processing so that signals are visible in all four sections of the detector in both images. The two images as presented therefore cannot be compared directly, i.e., the reference P47 quadrants in each image appear to have different brightnesses, even though they were in fact the same in the unprocessed images. However, even a cursory examination of the images reveals that the Exalite 404 screen exhibits a higher brightness than $\mathrm{P} 47$ at the same applied potentials. The data are presented more quantitatively in Figure 6, as a plot of the total signal recorded for a quadrant relative to the signal recorded for the $\mathrm{P} 47$ reference quadrant (maintained at a potential of $2.5 \mathrm{kV}$ ) as a function of the acceleration potential between the back face of the MCPs and the screen. The applied potential on the back MCP face was kept constant at $1.1 \mathrm{kV}$ for all measurements. The relative emission efficiency curves for P47, Exalite 389, and Exalite 404 screens shown in Figure 6 reveal that over the voltage range studied, Exalite 389 is less efficient than P47 by approximately a factor of two, while Exalite 404 is more efficient by a factor of two or three. It was not possible to continue these measurements to higher acceleration potentials due to the risk of electrical arcing across the gap between the MCPs and the screen.

We note that any quantitative conclusions relating to the relative brightness of the Exalite and P47 screens are valid only for P47 screens fabricated using our sedimentation protocol. P47 screens are also produced commercially using a brushing method, ${ }^{34}$ and P47 scintillators are also widely used in single crystal form. ${ }^{35-37}$ It has been well established by others that scintillator performance depends on particle size $e^{38}$ and layer thickness, ${ }^{39}$ as well as on the incident particle energy. However, in comparisons of an in-house built detector employing an Exalite 404 screen with a commercial ion detector employing a P47 screen (see Sec. III E), observed signals were much higher for the former, in line with the results reported above. 
(a)

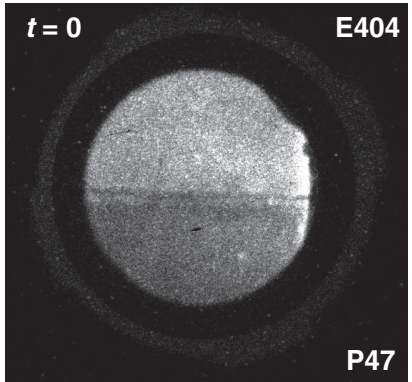

(b)

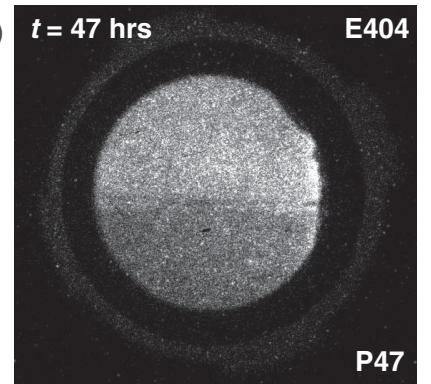

(c)

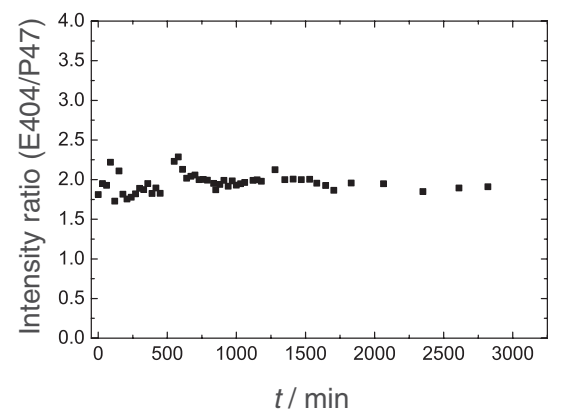

FIG. 7. Electron images recorded at (a) the start and (b) the end of the stability test period, using a split detector consisting of Exalite 404 (E404, top) and P47 (bottom) scintillators; (c) ratio of the total signal recorded for the Exalite 404 and P47 screens, as a function of time.

\section{Stability}

The most promising of the new scintillators, Exalite 404, was subjected to stability tests in which a screen was illuminated uniformly with electrons at a rate of around $3 \times 10^{9}$ electrons per second over a period of $47 \mathrm{~h}$. The experiment was carried out using a split screen combining a P47 and Exalite 404 scintillator, in order to allow a direct comparison of the performance of the Exalite screen with P47 over the timeframe of interest. The results of these measurements are shown in Figure 7. Figures 7(a) and 7(b) show images acquired at the start and end of the acquisition period, and Figure 7(c) shows a plot of the total signal intensity recorded on the Exalite screen relative to $\mathrm{P} 47$ as a function of time. The Exalite 404 screen shows a constant emission with an intensity around twice that of the P47 reference, with no observed degradation in signal.

One might perhaps expect organic scintillators to show rapid degradation under continued bombardment with high energy $(\sim 1.4 \mathrm{keV})$ electrons. However, the total amount of energy deposited into the scintillator screens per unit time under typical operating conditions is five or six orders of magnitude less than that deposited into the same compounds when employed in solution in a dye laser, so irreversible damage to the molecular constituents of the screens is likely to occur only over a relatively long timescale. This is consistent with the fact that we have not observed degradation in the performance of the screens, even when used over a timescale of several weeks in velocity-map imaging and imaging mass spectrometry experiments.

\section{E. Imaging performance}

To complete our study, the new Exalite 404 ion detector and a commercial P47 ion detector have been used to record photofragment velocity-map ion images of $\mathrm{CO}(J$ $=50$ ) photofragments formed in the $230 \mathrm{~nm}$ photolysis of OCS. Details of the fragmentation process can be found in Refs. 40-44.

The top panel of Figure 8 shows images acquired at three different sets of velocity-mapping potentials using the commercial P47 detector, operated with $1.5 \mathrm{kV}$ across the MCPs and $3.5 \mathrm{kV}$ on the phosphor screen. The bottom panel shows images acquired with the Exalite 404 detector, with $1.3 \mathrm{kV}$ across the MCPs and $3.0 \mathrm{kV}$ on the scintillator screen. Small differences in apparent image size are attributed to the fact that in the commercial detector, the phosphor is coated onto the inside of a fibre taper which forms the vacuum interface, while in our detector the phosphor is coated onto the internal surface of an ITO plate which forms the vacuum interface. In addition to any magnification caused by the fibre taper, the different focal planes (on the outside and inside surfaces of the vacuum interface window, respectively), lead to slightly different focusing conditions for the CCD camera used to image the two screens.

The higher brightness of the Exalite 404 screens relative to P47 means that the new imaging detector can generally be run at lower gain to achieve the same signal levels, thereby extending the useful lifetime of the microchannel plates. ${ }^{45}$ The two detectors yield images of comparable quality in terms of spatial resolution. Figure 9 compares the radial profiles (velocity distributions) extracted from the two images. The widths of the two peaks are very similar. The peak arising from the weak outer ring in the images, resulting from photolysis of vibrationally excited parent molecules, appears somewhat more intense in the data set recorded with the Exalite detector. However, since the images were recorded in two separate experiments involving a change of detector, this

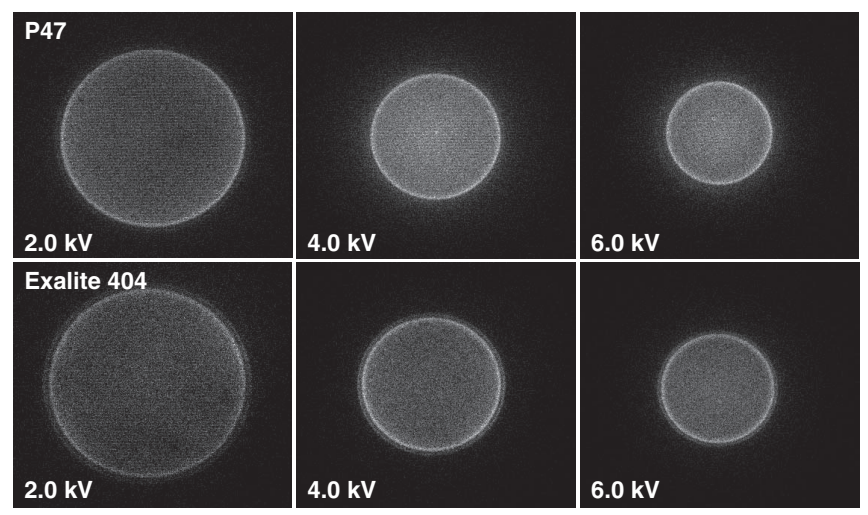

FIG. 8. Velocity map ion images of the $\mathrm{CO}(J=50)$ fragment formed in the $230 \mathrm{~nm}$ photodissociation of OCS. The series of ion images shown in the top row were collected with a commercial P47 detector, while the ones in the lower part of the figure were recorded with the new Exalite 404 screen. The potentials applied to the repeller plate of the velocity-map imaging ion optics are indicated for each image. 


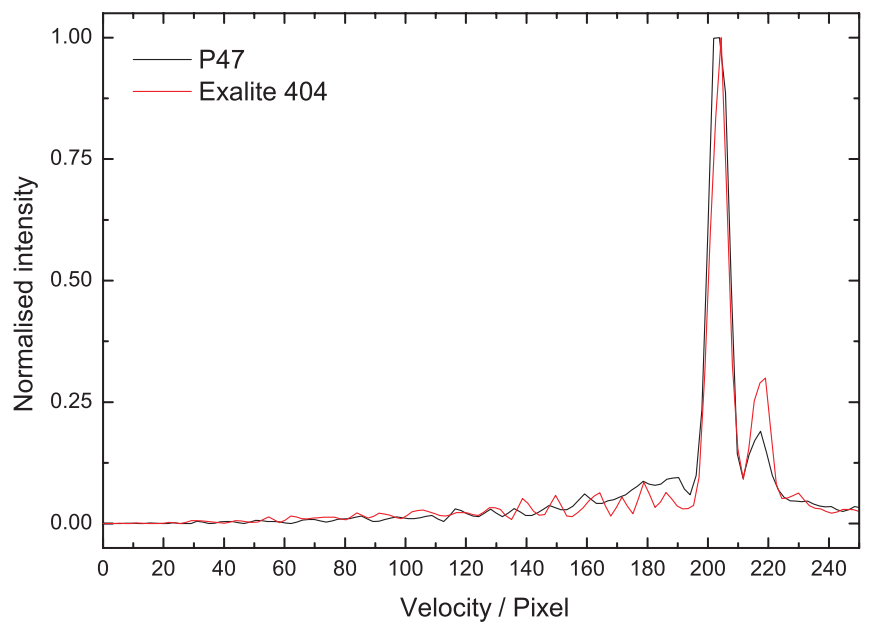

FIG. 9. Radial (velocity) distributions, in pixel units, obtained from the CO ion images shown in Figure 8.

could have various origins, including slightly different conditions within the molecular beam or a small degree of signal saturation of the inner ring when employing the brighter Exalite screen.

\section{SUMMARY AND CONCLUSIONS}

We have investigated a series of Exalite laser dyes as potential scintillator screen materials for use in velocity-map imaging, imaging mass spectrometry, and other charged particle imaging applications. Several dyes have been characterised in detail, and the most promising, Exalite 404, has been employed in a series of comparisons with a standard P47 phosphor (see Table III for a summary of the results). A detector employing the new Exalite 404 scintillator material shows improved time resolution and brightness, and comparable spatial resolution relative to a commercial P47-based detector. The emission spectrum is similar to that of P47, and no signal degradation has been observed over extended periods of operation. Fluorescent dye scintillator screens show considerable promise in applications for which a fast time response and efficient electron-to-photon conversion is desired, with the additional advantage that MCP-based particle detectors employing the new scintillators can be run at lower gain, potentially extending MCP lifetime.

TABLE III. Characteristics of Exalite 404, P47, and P43 scintillator screens. The data for the Exalite 404 and P47 detector were obtained in the experiments listed above, while the specifications for the P43 screen were taken from the Hamamatsu MCP guide. ${ }^{46}$

\begin{tabular}{lccc}
\hline \hline Property & Exalite 404 & P47 & P43 \\
\hline Emission lifetime & $3.7 \pm 0.2 \mathrm{~ns}$ & $69.3 \pm 0.8 \mathrm{~ns}$ & $>1 \mathrm{~ms}$ \\
Decay to 10\% & $7.7 \pm 0.3 \mathrm{~ns}$ & $168 \pm 2.3 \mathrm{~ns}$ & $>1 \mathrm{~ms}$ \\
Emission range (nm) & $380-550$ & $380-560$ & $450-675$ \\
Peak emission (nm) & 412 & 435 & 545 \\
Relative brightness & 1.97 & 1.00 & 3.33 \\
\hline \hline
\end{tabular}

\section{ACKNOWLEDGMENTS}

The support of the Engineering and Physical Sciences Research Council (U.K.) EPSRC(GB) via Programme Grant No. EP/G00224X/1 (to M.B. and C.V.), the European Union (EU) through Grant No. FP7 ITN "ICONIC" (Project Grant No. 238671 to M.B. and C.V.), STFC through a PNPAS award to M.B. and C.V., ERC through Starting Independent Researcher grant "ImageMS" (C.V.), and a proof of concept grant from ISIS Innovation Ltd. (M.B. and C.V.) are gratefully acknowledged. This work is subject to a patent application by ISIS Innovations Ltd. (UK Patent No.: 1310476.5).

${ }^{1}$ W. Crookes, Chem. News 87, 241 (1903).

${ }^{2}$ G. F. Knoll, Radiation Detection and Measurement, 4 th ed. (John Wiley \& Sons, Inc., 2010).

${ }^{3}$ J. N. Bull, J. W. L. Lee, S. H. Gardiner, and C. Vallance, "An introduction to velocity-map imaging mass spectrometry (VMImS)," Eur. J. Mass Spectrom. (published online).

${ }^{4}$ B. Winter, E. Halford, and M. Brouard, Int. J. Mass Spectrom. 356, 14 (2013).

${ }^{5}$ J. Aoki, H. Hazama, and M. Toyoda, J. Mass Spectrom. Soc. Jpn. 59, 57 (2011).

${ }^{6}$ J. H. Jungmann, L. MacAleese, R. Buijs, F. Giskes, A. de Snaijer, J. Visser, J. Visschers, M. J. J. Vrakking, and R. M. A. Heeren, J. Am. Soc. Mass Spectrom. 21, 2023 (2010).

${ }^{7}$ J. H. Jungmann, L. MacAleese, J. Visser, M. J. J. Vrakking, and R. M. A. Heeren, Anal. Chem. 83, 7888 (2011).

${ }^{8}$ J. H. Jungmann, D. F. Smith, L. MacAleese, I. Klinkert, J. Visser, and R. M. A. Heeren, J. Am. Soc. Mass Spectrom. 23, 1679 (2012).

${ }^{9}$ J. H. Jungmann and R. M. A. Heeren, Rapid Commun. Mass Spectrom. 27, 1 (2013).

${ }^{10}$ M. Froesch, S. L. Luxembourg, D. Verheijde, and R. M. A. Heeren, Eur. J. Mass Spectrom. 16, 35 (2010).

${ }^{11}$ M. Hayashi and Y. Naito, J. Mass Spectrom. Soc. Jpn. 59, 23 (2011).

${ }^{12}$ A. Nomerotski, S. Adigun-Boaye, M. Brouard, E. Campbell, A. Clark, J. Crooks, J. J. John, A. J. Johnsen, C. Slater, R. Turchetta et al., Nucl. Instrum. Methods Phys. Res. A 633, S243 (2011).

${ }^{13}$ M. Brouard, E. Halford, A. Lauer, C. S. Slater, B. Winter, W. H. Yuen, J. J. John, L. Hill, A. Nomerotski, A. Clark et al., Rev. Sci. Instrum. 83, 114101 (2012)

${ }^{14}$ A. T. Clark, J. P. Crooks, I. Sedgwick, R. Turchetta, J. W. L. Lee, J. J. John, E. S. Wilman, L. Hill, E. Halford, C. S. Slater et al., J. Phys. Chem. A 116, 10897 (2012).

${ }^{15}$ J. J. John, M. Brouard, A. Clark, J. Crooks, E. Halford, L. Hill, J. W. L. Lee, A. Nomerotski, R. Pisarczyk, I. Sedgwick et al., JINST 7, C08001 (2012).

${ }^{16}$ I. Sedgwick, A. T. Clark, J. P. Crooks, R. Turchetta, J. W. L. Lee, J. J. John, E. S. Wilman, L. Hill, R. Pisarczyj, E. Halford et al., in Proceedings of the New Circuits and Systems Conference (NEWCAS), 2012.

${ }^{17}$ F. Dubois, R. Knochenmuss, and R. Zenobi, Int. J. Mass Spectrom. Ion Process. 169-170, 89 (1997).

${ }^{18}$ F. Dubois, R. Knochenmuss, R. Zenobi, A. Brunelle, C. Deprun, and Y. L. Beyec, Rapid Commun. Mass Spectrom. 13, 786 (1999).

${ }^{19}$ F. Dubois, R. Knochenmuss, and R. Zenobi, Rapid Commun. Mass Spectrom. 13, 1958 (1999).

${ }^{20}$ W. R. Leo, Techniques for Nuclear and Particle Physics Experiments, 2nd ed. (Springer-Verlag, 1994).

${ }^{21}$ R. C. Sangster and J. W. Irvine, J. Chem. Phys. 24, 670 (1956).

${ }^{22}$ M. Rinke, H. Guesten, and H. J. Ache, J. Phys. Chem. 90, 2666 (1986).

${ }^{23}$ J. M. Kauffman, C. J. Kelley, A. Ghiorghis, E. Neister, L. Armstrong, and P. R. Prause, Laser Chem. 7, 343 (1987).

${ }^{24}$ D. J. Schneider, D. A. Landis, P. A. Fleitz, C. J. Seliskar, J. M. Kaufman, and R. N. Steppel, Laser Chem. 11, 49 (1991).

${ }^{25}$ J. M. Kauffman, C. J. Kelley, A. Ghiorghis, E. Neister, and L. Armstrong, Laser Chem. 8, 335 (1988).

${ }^{26}$ P. A. Fleitz, C. J. Seliskar, R. N. Steppel, J. M. Kauffman, C. J. Kelly, and A. Ghiorghis, Laser Chem. 11, 99 (1991).

${ }^{27}$ S. R. Inamdar, J. R. Mannekulta, B. G. Bulimani, and M. I. Savadatti, Chem. Phys. Lett. 429, 141 (2006).

${ }^{28}$ T. A. Merz, P. G. Waddell, and J. M. Cole, J. Phys. Chem. C 117, 8429 (2013). 
${ }^{29}$ J. R. Tymianski and J. K. Walker, U.S. patent 5606638A (December 26, 1995).

${ }^{30}$ W. S. Hopkins, L. M. Lipciuc, S. H. Gardiner, and C. Vallance, J. Chem. Phys. 135, 034308 (2011).

${ }^{31}$ A. H. Kung, J. F. Young, and S. E. Harris, Appl. Phys. Lett. 22, 301 (1973).

${ }^{32}$ Simion8.0, see http://www.simion.com.

${ }^{33}$ F. J. Duarte and L. Hillman, Dye Laser Principles (Academic Press Limited, London, 1990).

${ }^{34}$ W. M. Yen and M. J. Weber, Inorgnic Phosphors: Composition, Preparation, and Optical Properties (CRC Press, 2004), Vol. 1.

${ }^{35}$ M. Dahlbom, L. R. MacDonald, L. Eriksson, M. Paulus, M. Andreaco, M. E. Casey, and C. Moyers, IEEE Trans. Nucl. Sci. 44, 1114 (1997).

${ }^{36}$ M. Moszynski, M. Balcerzyk, M. Kapusta, D. Wolski, and C. L. Melcher, IEEE Trans. Nucl. Sci. 47, 1324 (2000).

${ }^{37}$ R. Lecomte, C. Pepin, D. Rouleau, A. Saoudi, M. S. Andreaco, M. Casey, R. Nutt, H. Dautet, and P. P. Webb, IEEE Trans. Nucl. Sci. 45, 478 (1998).
${ }^{38}$ R. Kingslake and B. J. Thompson, Applied Optics and Optical Engineering, 6th ed. (Academic Press, London, 1980).

${ }^{39}$ J. D. McGee, Photo-electronic Image Devices (Academic Press Limited, London, 1966), Vol. 22A.

${ }^{40}$ Y. Sato, Y. Matsumi, M. Kawasaki, K. Tsukiyama, and R. Bersohn, J. Phys. Chem. 99, 16307 (1995).

${ }^{41}$ M. Brouard, A. V. Green, F. Quadrini, and C. Vallance, J. Chem. Phys. 127, 084304 (2007)

${ }^{42}$ M. Brouard, F. Quadrini, and C. Vallance, J. Chem. Phys. 127, 084305 (2007)

${ }^{43}$ M. L. Lipciuc and M. H. M. Janssen, Phys. Chem. Chem. Phys. 8, 3007 (2006)

${ }^{44}$ M. L. Lipciuc and M. H. M. Janssen, J. Chem. Phys. 126, 194318 (2007).

${ }^{45}$ J. L. Wiza, Nucl. Instrum. Methods 162, 587 (1979).

${ }^{46}$ Hamamatsu, MCP ASSEMBLY, 2006. 